\title{
A GMPLS/OBS Network Architecture Enabling QoS-aware End-to-End Burst Transport
}

\author{
Pedro Pedroso*, Mirosław Klinkowski ${ }^{\dagger}$, Jordi Perelló*, Salvatore Spadaro*, Davide Careglio*, Josep Solé-Pareta* \\ *CCABA, Universitat Politècnica de Catalunya (UPC), Barcelona, Spain, e-mail: ppedroso@ac.upc.edu \\ ${ }^{\dagger}$ National Institute of Telecommunications (NIT), Wrocław, Poland, e-mail: mklinkow@itl.waw.pl
}

\begin{abstract}
This paper introduces a Generalized Multi-Protocol Label Switching (GMPLS)-enabled Optical Burst Switched (OBS) network architecture featuring end-to-end QoS-aware burst transport services. This is achieved by setting up burst Label Switched Paths (LSPs) properly dimensioned to match specific burst drop probability requirements. These burst LSPs are used for specific guaranteed QoS levels, whereas the remaining network capacity can be left for best-effort burst support. Aiming to ensure the requested burst drop probability figures even under bursty traffic patterns, burst LSPs' performance is continuously monitored. Therefore, GMPLS-driven capacity reconfigurations can be dynamically triggered whether unfavorable network conditions are detected. Through the paper, the GMPLS/OBS architecture is firstly detailed, followed by the presentation of the optimized methods used for the initial burst LSP dimensioning. The successful network performance is finally illustrated by simulations on several network scenarios.
\end{abstract}

\section{INTRODUCTION AND MOTIVATION}

Paving the way to the future Internet, all-optical networks have broken the limitations of Optical/Electronic/Optical (OEO) conversions in the network, which cannot match the transmission rates offered by recent advances in Dense Wavelength Division Multiplexing (DWDM) technology [1].

In this context, the Optical Burst Switching (OBS) paradigm leverages on the statistical multiplexing of optical data plane resources to enable sub-wavelength switching in the network [2]. This is achieved by aggregating the packets arriving at ingress nodes into bursts to be sent individually through a bufferless optical network. With aims to minimize the overhead introduced along the burst signaling process, OBS networks typically rely on one-way resource reservation. However, such on-the-fly resource reservation does not ensure successful burst delivery, as they could encounter contention at intermediate nodes and become lost. Looking at the literature, a large number of techniques have been proposed to reduce burst losses in OBS networks, mostly focused on providing intelligence to the optical layer. Examples of such techniques are, amongst many others, link congestion prediction [3][4], wavelength selection considering the streamline effect [5], feedback mechanisms to control network congestion and connection admission [6][7] or QoS-aware deflection routing [8]. Nevertheless, none of them guarantee specific QoS figures as the offered load to the network increases.

Conversely, Optical Circuit Switching [9] networks allow end-to-end QoS compliant optical circuits between source/destination nodes. For the sake of flexibility, these circuits can be dynamically provisioned by a control plane governing the underlying optical devices. To support these tasks, the IETF has defined Generalized Multi-Protocol Label Switching (GMPLS) as a set of protocols covering the required signaling, routing and management functionalities [10]. Nevertheless, GMPLS/OCS still provides very coarse granularity (a whole wavelength), being sub-wavelength switching only feasible through electrical grooming.

In this paper we introduce a GMPLS-enabled OBS network featuring QoS-guaranteed transport services. The rationale behind our proposal is to move the overall network intelligence to the GMPLS control plane, being the OBS layer only responsible for local contention resolution. To this end, an extended GMPLS control plane is placed on top of the OBS layer, providing this one with burst LSPs provisioning capabilities. These burst LSPs are tailored to specific QoS requirements, guaranteeing the requested service levels.

The contribution of this work is three-fold. First of all, we define the GMPLS/OBS network architecture, detailing the behavior of its control and transport planes (Section II). Second, we focus on the offline burst LSP configuration problem in the GMPLS/OBS network by proposing a Mixed Integer Linear Programming (MILP) model (Section III). Third, we consider situations where unexpected traffic peaks or highly dynamic patterns may appear and negatively impact on the provided QoS levels. In this context, we propose burst LSP performance monitoring mechanisms able detect undesired traffic surges and trigger burst LSP reconfiguration procedures when needed (Section IV). The performance of these contributions are finally evaluated through simulations on different network scenarios (Section V).

\section{GMPLS/OBS NETWORK ARCHITECTURE}

In the proposed GMPLS/OBS network architecture, the extended GMPLS control plane lies on top as the actual OBS network controller, setting up, maintaining, reconfiguring and tearing down burst LSPs (hereafter simply referred as LSPs) according to the client traffic demands and QoS requirements. While the OBS control layer must share the same physical network as the OBS data plane due to the strict time relation between Burst Control Packets (BCPs) and optical bursts, the GMPLS control plane can follow a different topology than the latter, even supported on a separated network.

As mentioned before, the main benefit of OBS is the statistical multiplexing, better exploiting those underlying data plane resources. However, due to the lack of optical buffering, 


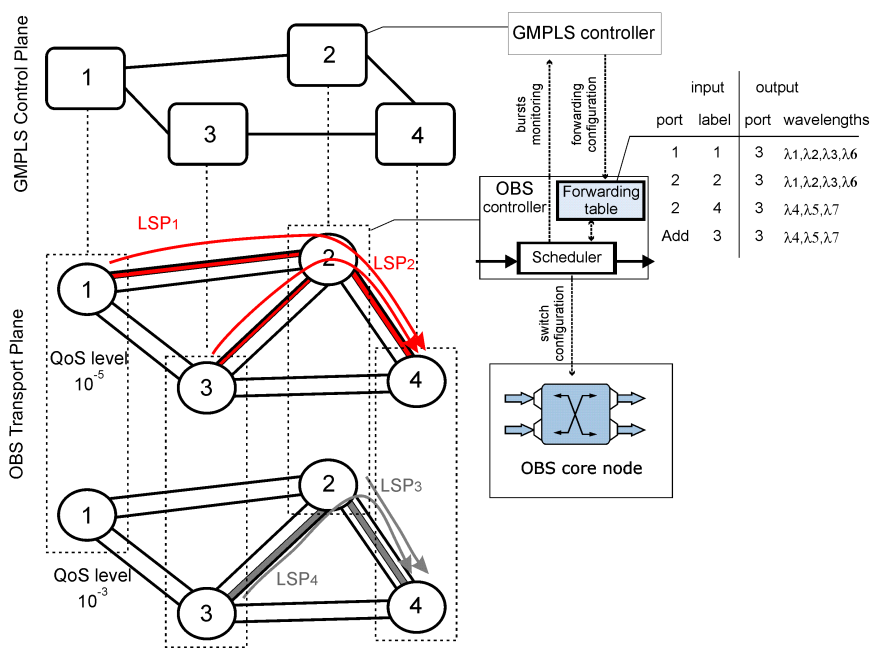

Fig. 1. Virtual topologies in GMPLS/OBS network.

random burst losses can be experienced in OBS due to contention. In our GMPLS/OBS network architecture, the burst loss probability (BLP) is controlled by tightly dimensioning those LSPs supporting burst data traffic.

In particular, a virtual topology (VT) of LSPs is deployed for each offered QoS level, where all LSPs share a large enough number of wavelengths per link so that the requested end-to-end QoS can be provided. Note that wavelength sharing, which fosters the statistical multiplexing of network resources, is limited to only those LSPs with the same QoS level. Otherwise, OBS nodes would have to be provided with complex scheduling algorithms to enable QoS level differentiation over the same set of output wavelengths.

In Fig. 1, an example of a GMPLS/OBS network configured with two virtual topologies and enabling two QoS levels is illustrated. On the right hand side of the figure, we can observe the GMPLS controller of node 2, in charge of configuring the forwarding table of the OBS controller that configures the optical switch by means of the scheduler. Four LSP crossing node 2 are currently established in the network, two per each virtual topology. Four entries are hence configured in the forwarding table. While all LSPs use the same output port 3, only those LSPs with the same QoS share output wavelengths; bursts belonging to $L S P_{1}$ and $L S P_{2}$ can leave node 2 using either $\lambda_{1}, \lambda_{2}, \lambda_{3}$, or $\lambda_{6}$ while those belonging to $L S P_{3}$ and $L S P_{4}$ only $\lambda_{4}, \lambda_{5}$, or $\lambda_{7}$.

For the sake of simplicity, only two service classes are considered in this work, namely, a High-Priority (HP) with guaranteed QoS level and a Best Effort (BE) class. Therefore, only a single virtual topology of LSPs for the HP class has to be dimensioned in the network, restricting the BE class to use the spare network capacity. Note, however, that multiple VTs can be dimensioned with the model in Section III.

This GMPLS/OBS architecture aims at keeping the OBS layer as fast as possible, as only simple local decisions are required (e.g., select a wavelength among a set of pre-selected ones). In fact, virtual topology set up and reconfiguration

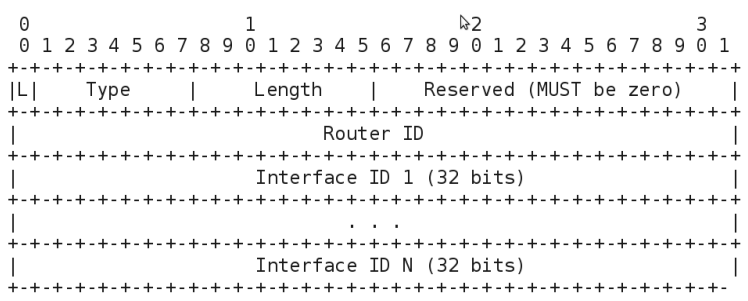

Fig. 2. Proposed extensions to the RSVP-TE Unnumbered interface ID subobject for burst LSP signaling.

actions (e.g., due to undesired link congestion or link failures) are moved to the GMPLS-enabled control plane. To this goal, the standard GMPLS protocol set has to be extended accordingly. This section introduces the extensions to GMPLS RSVP-TE protocol required for the setup of the QoS-aware LSPs resulting from the dimensioning models in section III. Moreover, section IV presents the procedures at the GMPLS node to adjust their capacity dynamically upon congestion. Due to the lack of space, however, we leave for future work those procedures and GMPLS protocol extensions involved in online LSP provisioning (i.e., LSPs supporting new demands not contemplated in the original traffic matrix).

In standard RSVP-TE [11], explicit routing is achieved by means of an EXPLICIT_ROUTE object in Path messages. This object encapsulates a list of sub-objects determining the nodes and links along the explicit route. In case of unnumbered links [12], Unnumbered interface ID sub-objects contain, for each traversed node, the router IP address (Router ID) and the identifier of the interface associated to the desired output link (e.g., wavelength). This allows only one wavelength per hop to be allocated with standard RSVP-TE. As mentioned before, however, burst LSPs may bundle several wavelengths per hop. Fig. 2 presents the extensions to Unnumbered interface ID subobject for burst LSP signaling. In this case, the Router ID field is followed by the ID's of the interfaces associated to each wavelength to be allocated for the LSP on the downstream link. Departing from the network characteristics and the static traffic matrix, the following section presents the dimensioning model to obtain the explicit routes and wavelengths for all LSPs composing the targeted set of VTs.

\section{MILP MODEL FOR LSP DIMENSIONING}

In order to guarantee certain level of QoS in terms of burst losses, wavelength resources have to be dimensioned properly. In this section, we address the problem of the VT design that concerns the establishment of explicit LSPs (also referred as paths in this section) and the allocation of wavelengths in network links to support connections with QoS guarantees. More specifically, we are looking for such network routing that for given set of (long-term) traffic demands and endto-end requirements on the burst loss rate minimizes the overall number of allocated wavelengths (i.e., the wavelength usage) in the network. To treat the problem of absolute QoS guarantees analytically, we employ the non-reduced load approximation [13] of a common OBS network loss 
model [14]. Modelling assumptions are then represented as a set of constraints in a Mixed Integer Liner Programming (MILP) formulation.

Due to the space limitations, the main modelling steps are briefly presented in this paper. For more details as well as some heuristics for the VT design problem we refer to [15].

\section{A. Notation}

We use $\mathcal{G}=(\mathcal{V}, \mathcal{E})$ to denote the graph of an OBS network; the set of nodes is denoted as $\mathcal{V}$, and the set of unidirectional links is denoted as $\mathcal{E}$. Link $e \in \mathcal{E}$ comprises $W_{e}$ wavelengths.

Let $\mathcal{P}$ denote the set of predefined candidate LSPs between source $s$ and termination $t$ nodes, $s, t \in \mathcal{V}$, and $s \neq t$. Each path $p \in \mathcal{P}$ is identified with a subset $p \subseteq \mathcal{E}$. Adequately, subset $\mathcal{P}_{e} \subseteq \mathcal{P}$ identifies all paths that go through link $e$. Let $\delta=\max \left\{\delta_{p}: p \in \mathcal{P}\right\}$ be the length of the longest path in the network, where $\delta_{p}$ is the length (in hops) of path $p$.

Let $\mathcal{D}$ denote the set of demands with QoS guarantees, where each demand corresponds to a pair of sourcetermination nodes. For each demand $d \in \mathcal{D}, h_{d} \in \mathbb{R}_{+}$denotes the volume of traffic; for convenience, $h_{p}=h_{d}$ for $p \in \mathcal{P}_{d}$.

Let $\mathcal{P}_{d} \subseteq \mathcal{P}$ denote the set of candidate LSPs supporting demand $d ; \mathcal{P}=\bigcup_{d \in D} \mathcal{P}_{d}$. Each subset $\mathcal{P}_{d}$ comprises a (small) number of paths, e.g., $k$ shortest paths, and a burst can follow one of them.

\section{B. Modelling Assumptions}

1) Routing: The network applies source-based routing. The selection of path $p$ from set $\mathcal{P}_{d}$ is performed according to a decision variable $x_{p}$ (also referred to as the routing variable). We assume unsplittable routing, in particular, a burst flow is routed over path $p$ iff $x_{p}=1$ and there is only one path $p \in \mathcal{P}_{d}$ such that $x_{p}=1$. Accordingly, traffic $\rho_{p}$ offered to path $p \in \mathcal{P}_{d}$ is calculated as $\rho_{p}=x_{p} h_{d}$.

2) Burst Losses: Due to the complexity of the Erlang fixedpoint computation in the common OBS network loss model [14], we assume a simplified model based on the non-reduced load calculation [13]. In this model, to estimate traffic load $\rho_{e}$ offered to link $e$, we add up the traffic load $\rho_{p}$ offered to each path $p \in \mathcal{P}$ that crosses this link: $\rho_{e}=\sum_{p \in \mathcal{P}: p \ni e} \rho_{p}=$ $\sum_{p \in \mathcal{P}: p \ni e} x_{p} h_{p}, e \in \mathcal{E}$. The use of such approximation is justified by its accuracy, particularly under low overall burst losses (below $10^{-2}$ ) [13].

Moreover, we take the common assumption in the literature of i.e.d. burst arrivals, i.i.d. burst durations, together with the assumption of the full wavelength conversion capability in network nodes. Accordingly, the Erlang B-loss formula $\mathcal{B}(\rho, w)$ is used to model the probability $B_{e}$ that a burst is lost in link $e$.

3) Burst Loss Guarantees: We assume each demand belonging to a QoS class has the same end-to-end (e2e) burst loss probability $B^{e 2 e}$ requirements. To meet the goal of the e2e QoS for each demand $d \in \mathcal{D}$, we assume that at each link the burst losses are kept below certain level $B^{\text {link }}$, i.e., $B_{e} \leq B^{l i n k}, \forall e \in \mathcal{E}$. For the rest of the paper, we consider $B^{\text {link }}$ fixed, the same for each link, and determined according to $B^{\text {link }}=1-\left(1-B^{e 2 e}\right)^{1 / \delta}$. This model is a common model frequently used to assure QoS guarantees in loss networks and it is also applicable under unsplittable source routing in OBS [15].

4) Wavelength Allocation: We consider each QoS class has a number of wavelengths allocated in network links which are not shared with other QoS classes. Although, in this paper, we focus on a single QoS class, still the restricted approach allows to extend the model easily to the scenario with multiple QoS classes.

The last modelling step is to define a dimensioning function $F_{e}(\cdot)$ which for given traffic load $\rho_{e}$ determines the minimum number of wavelengths to be allocated in link $e$ so that to satisfy the blocking $B^{l i n k}$ requirements. Such a function is given by a discrete (discontinuous, step-increasing) link dimensioning function $F_{e}\left(\rho_{e}\right)=\left[\mathcal{B}^{-1}\left(\rho_{e}, B^{\text {link }}\right)\right]$, where $\mathcal{B}^{-1}\left(\rho_{e}, B^{\text {link }}\right)$ is the inverse of the Erlang B Loss formula extended to the real domain [16], and $\lceil\cdot\rceil$ is the ceiling function.

\section{Problem Formulation}

It is convenient to define $a_{w}$ as the maximal load supported by $w$ wavelengths given target blocking probability $B^{l i n k}$, i.e., $a_{w}=\mathcal{B}^{-1}\left(w, B^{\text {link }}\right)$. Although there is no close formula to calculate $\mathcal{B}^{-1}$, still we can use a line search method (see e.g., [17]) to find the root $\rho^{*}$ of function $f(\rho)=$ $B^{\text {link }}-\mathcal{B}(\rho, w)$ so that to approximate the value of $a_{w}$ by $a_{w}=\rho^{*}$ for each $w \leq \max \left\{W_{e}: e \in \mathcal{E}\right\}$. Also, we introduce a segmentation on load segments: $b_{w}=a_{w}-a_{w-1}, w=$ 1.. $\max \left\{W_{e}: e \in \mathcal{E}\right\}$.

Finally, we substitute $F_{e}(\cdot)$ with its piecewise linear approximation, $F_{e}\left(\rho_{e}\right)=\min \left\{w: a_{w} \geq \rho_{e}\right\}$, which further allows us to express the dimensioning function by means of a $0-1$ integer programming (IP) formulation [13]. This formulation makes use of a set of binary variables $\left\{u_{e}^{w}: e \in \mathcal{E}, w=\right.$ $\left.1 . . W_{e}\right\} ; u_{e}^{w}$ is active iff $w$ or more wavelengths are allocated in link $e$.

Our VT design problem can be formulated as a MILP problem:

$$
\begin{aligned}
& \text { minimize } \quad \sum_{e} \sum_{w} u_{e}^{w} \\
& \text { subject to } \\
& \sum_{p \in \mathcal{P}_{d}} x_{p}=1, \quad \forall d \in \mathcal{D}, \\
& \sum_{p \in \mathcal{P}: p \ni e} h_{p} x_{p}-\rho_{e}=0, \quad \forall e \in \mathcal{E}, \\
& \rho_{e} \leq a_{W_{e}}, \quad \forall e \in \mathcal{E}, \\
& \sum_{w=1 . . W_{e}} u_{e}^{w} b_{w}-\rho_{e} \geq 0, \quad \forall e \in \mathcal{E}, \\
& u_{e}^{w}-u_{e}^{w+1} \geq 0, \quad \forall e \in \mathcal{E}, w=1 . . W_{e}-1, \\
& u_{e}^{w} \in\{0,1\}, \quad \forall e \in \mathcal{E}, w=1 . . W_{e}, \\
& \bar{x} \in\{0,1\}^{|\mathcal{P}|}, \bar{\rho} \in \mathbb{R}_{+}^{|\mathcal{E}|},
\end{aligned}
$$

where $\rho_{e}$ is an auxiliary variable representing load in link $e$.

The objective of the optimization problem is to minimize the total number of wavelengths utilized in the network. (1a) 
are the routing constraints. (1b) are auxiliary constraints of the non-reduced load calculation. (1c) are the link capacity constraints. (1d) and (1e) result from the 0-1 representation of function $F_{e}(\cdot)$. In particular, the number of wavelengths in link $e$ should be such that the maximum traffic load it can support (calculated as the sum of active load segments $b_{w}$ ) is greater or equal to offered traffic load $\rho_{e}$. Besides, (1e) are ordering constraints, i.e., if $w$ wavelengths are utilized so $w-1$ wavelengths are utilized as well. Finally, (1f) and (1g) are the variable range constraints.

Note that (MILP) is a variant of the well-known discrete cost multicommodity flow problem (DCMCF) which is a difficult problem [18]. Still, performed experiments show that a good sub-optimal solution (optimality gap below $2 \%$ ) can be found in reasonable time (from several to some hundreds of seconds) for a 28-node network using the CPLEX v.11.1 solver [15].

\section{DYNAMIC LSP CAPACITY RECONFIGURATION}

In highly dynamic networks, such as OBS networks, the volume of offered traffic may change abruptly and unexpectedly. In these scenarios, the previous offline VT dimensioning would be inefficient only by itself. In the proposed GMPLS/OBS architecture, we also deploy an auxiliary mechanism to induce a dynamic character to the transport network.

The devised mechanism operates in a proactive manner avoiding LSPs to reach either full capacity, which would increase the number of dropped bursts, or an inefficient use of wavelengths. The decision to increase/decrease the number of wavelengths associated to a LSP can be taken either on a local or end-to-end basis approach, spanning n-hops. Here, only locally based decisions spanning 1 single hop are considered, leaving for further work end-to-end alternatives. Specifically, the devised dynamic LSP capacity reconfiguration (DLCR) mechanism works as follows.

Each OBS node $n \in \mathcal{V}$ is responsible for monitoring the HP traffic being offered by all LSPs supported on any of its output links $i, i=\{1, . . \operatorname{deg}(n)\}$, over a sliding temporal window $T$ with duration $|T|$. Note that by considering only one highpriority class, the LSPs share the same set of wavelengths at each output link, facing the same wavelength occupancy. Therefore, the offered high-priority traffic load to an output link $i$ in the node can be expressed as:

$$
\rho_{(i)}=\frac{\sum_{b \in B} t_{b}}{|T|}
$$

where $t_{b}$ is the duration of the incoming burst $b \in B, B$ denotes all the incoming HP bursts to be switched at node $n$ within $|T|$.

At every monitoring interval, the OBS controller sends a trap message to its respective GMPLS controller reporting the current HP traffic being offered to its output links. Upon reception, the GMPLS controller is then responsible for detecting sudden traffic changes and triggering LSP reconfiguration if required. Given $\rho_{(i)}$, it verifies whether the LSPs' size at link $i, L_{i}$ (i.e. the number of wavelengths), is still appropriate. To this end, it estimates the current HP traffic BLP using the Erlang-B loss formula $(\mathcal{B})$ and checks if the value remains below the demanded QoS threshold $\Omega$. For this, the following assessment condition is verified:

$$
e B L P_{H P}=\mathcal{B}\left(\rho_{(i)}, L_{i}\right)<\Omega \quad \forall_{i}
$$

The DLCR mechanism does not trigger the reconfiguration request before $W$ consecutive windows with $e B L P_{H P} \geq \Omega$ (per each output link $i$ ). Such decision helps to maintain the stability of the system by remaining insensitive to short-term traffic changes. If $W$ is reached, however, the traffic peak is considered as significant and a 1-hop LSP expansion is triggered. To this goal, GMPLS computes the new set of wavelengths for the LSPs on output link $i$ to properly face the measured HP traffic load. Such set of additional wavelengths is given by: $\phi(i)=\mathcal{B}^{-1}\left(\rho_{(i)}, \Omega\right)-L_{i}$ where $\mathcal{B}^{-1}\left(\rho_{(i)}, \Omega\right)$ returns the number of wavelengths needed to satisfy $\Omega$ for the new estimated traffic.

On the other hand, GMPLS can verify that the capacity of the LSP is over-dimensioned (i.e. $\phi(i)<0$ ). In such a case, a given number of wavelengths may be released.

From the GMPLS control plane perspective, the rearrangement of those wavelengths assigned to the downstream link of a given LSP is quite straightforward. In particular, the RSVPTE module in the GMPLS controller records the information of all configured LSPs in the node following the Path State Block (PSB) structure defined in [19][20]. For each LSP, the information contained in the PSB describes a similar structure than the Path message that originally signaled it, namely, a Session, a Sender Template and an ERO object. As previously detailed in Figure 2, the Unnumbered interface ID sub-objects in the Path message ERO has been here extended so that multiple wavelengths can be allocated at every LSP hop. Therefore, the allocation of additional wavelengths to a certain LSP is as easy as including in the specific Unnumbered interface ID sub-object those interface IDs associated to the wavelengths. Conversely, if some wavelengths already associated to the LSP would have to be released, the associated interface IDs would be removed from the specific Unnumbered interface ID subobject. In this way, assuming that the LSP would have to be released, that information in the PSB would be used to deallocate the resources supporting it.

\section{Performance Assessment}

Simulations are performed to estimate the performance of the proposed QoS-aware GMPLS/OBS architecture under different network scenarios and conditions. The objective is to provide a validation of both i) planning strategies as the offline VT dimensioning and of ii) operational strategies like the proactive reaction to unexpected traffic peaks. The simulations are executed on the ad-hoc JAVOBS simulator developed in [21], here extended to implement the proposed architecture.

\section{A. Simulation scenarios}

Two reference network scenarios have been used in order to claim for topology independence, namely, the 14-node 

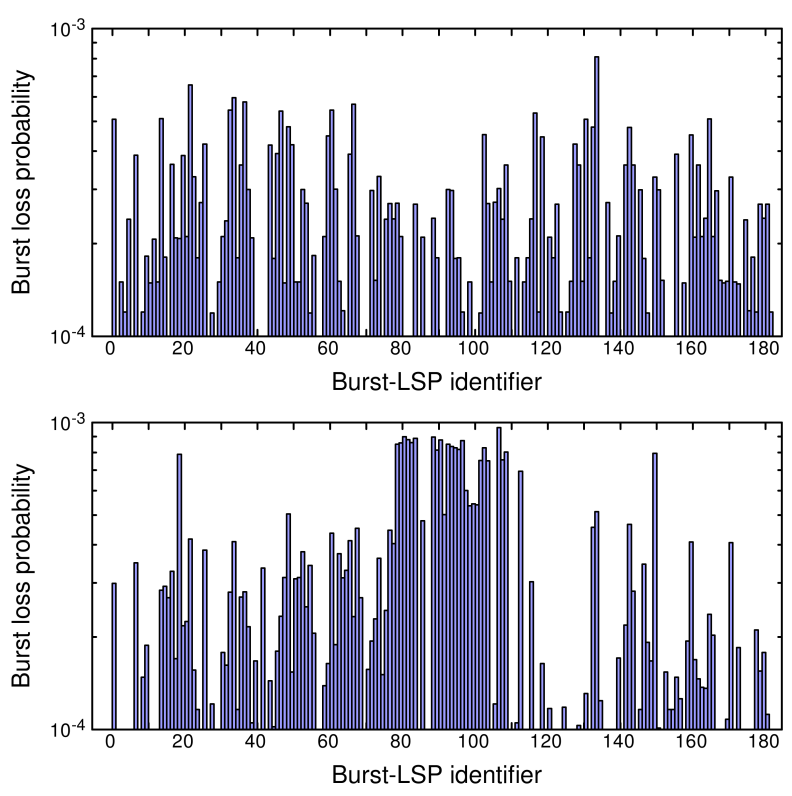

Fig. 3. HP burst losses with uniform (top) and non-uniform (bottom) traffic.

NSFNET and the 28-node EON network, where all links support 32 bidirectional wavelengths at $10 \mathrm{Gbps}$. Regarding the traffic characteristics, the $[75 \%-25 \%]$ HP-BE traffic ratio is assumed for the whole set of simulations in all network scenarios. All network nodes generate traffic following a Poisson process, based on either uniform or non-uniform traffic matrices. In particular, the HP traffic requires a BLP $<10^{-3}$ and is routed throughout a full-mesh VT of LSPs. Therefore, there is $N *(N-1)$ LSPs in each network scenario. Conversely, the BE traffic is routed using the Dijkstra's Shortest Path algorithm. In the network, GMPLS/OBS nodes are equipped with full wavelength conversion capabilities, a GMPLS control node, a traffic-engineered (TE) database to store LSP related information and an LSP occupancy monitoring system. Neither deflection or preemption mechanisms are initially considered on the experiments.

\section{B. Validation of the VT dimensioning model}

Fig. 3 shows the BLP of all LSPs in the 14-node NSFNET network, where a total load of 0.5 is offered according to uniform (top) and non-uniform (bottom) traffic demands. The main objective here is to validate the VT dimensioning model, ensuring that the requested QoS constraints in terms of BLP are finally meet in every established LSP. As seen, the experienced BLP in all individual LSPs is below the demanded BLP $<10^{-3}$ for both uniform and non-uniform traffic distributions. This not only validates the proposed dimensioning model, but also shows that it behaves independently of the traffic distribution in the network.

\section{Comparison of GMPLS/OBS against conventional OBS}

Figs. 4 and 5 compare the proposed GMPLS/OBS architecture against a conventional OBS architecture (convOBS). The results have been extracted from both NSFNET and
EON network scenarios, where a uniform traffic matrix is considered. In convOBS, deflection routing is applied for the HP class bursts as a QoS differentiation mechanism. From both figures, we observe an outstanding behavior of the proposed GMPLS/OBS architecture. As seen, the BLP of the HP traffic remains constant in the entire offered load range in both NSFNET and EON network scenarios, being considerably below the demanded QoS threshold $\left(10^{-3}\right)$. In contrast, the BLP of the HP traffic grows exponentially with the offered load to the network in convOBS, being soon over the BLP threshold in the NSFNET network (from an offered load equal to 0.45 ) and never reaching it in the EON case. For example, differences around two orders of magnitude can be observed in the NSFNET network scenario for an offered load of 0.8 .

Focusing on the BE traffic transmission, we have similar BLP values in both GMPLS/OBS and convOBS architectures, slightly higher in the former. The performance of the BE class can be further improved when applying deflection routing or the burst preemption mechanism in the network. Such scenarios are left for further study. However, we shall mention that the main concern here is to guarantee the QoS for HP traffic, as agreed with potential OBS network clients through Service Level Agreements (SLAs).

\section{Assessment of the dynamic LSP reconfiguration}

In order to evaluate the dynamic LSP reconfiguration mechanism proposed in Section IV, we enforce a peak of HP traffic of limited duration in a LSP. This leads to the following actions: i) expansion of the LSP and ii) return to original LSP configuration. Fig. 6 shows a sample of the execution time during which we monitor the traffic occupancy of an output link of the NSFNET network. The QoS control is performed in terms of HP burst loss probability (eBLP), which is estimated every $T$ window with $|T|=5 \mathrm{~ms}$. From the figure, we observe that as soon as the traffic peak occurs, around $t=30 \mathrm{~ms}$ (the offered load goes from 4,2 Er. to 8.5Er.), the GMPLS instance detects it and remains on a holding stage during $W=5$ consecutive $T$ windows (i.e. $25 \mathrm{~ms}$ ) with an eBLP above the QoS level. Following the process as described in Section IV, those interface IDs of each additional wavelength are included in the specific Unnumbered interface ID subobject maintained in the PSB at the RSVP-TE module of the GMPLS controller. Once the PSB is updated, the OBS controller is notified in order to update its forwarding table and the LSPs acquire extended properties. As a result, the eBLP returns to values below the QoS threshold. At approx. $400 \mathrm{~ms}$, however, the offered load is reduced back to its previous value of 4.2Er (the traffic peak ends), so that the LSP becomes now over-dimensioned for the current offered load. Therefore, in order to achieve good resource usage, a counterpart process is triggered. After $W=5$ consecutive $T$ windows with extra allocated resources (i.e. wavelengths), the LSPs return to their initial configuration.

This DLCR mechanism induces a dynamic character (throughout GMPLS) to the proposed architecture to properly handle unexpected traffic demand surges. It is worth men- 


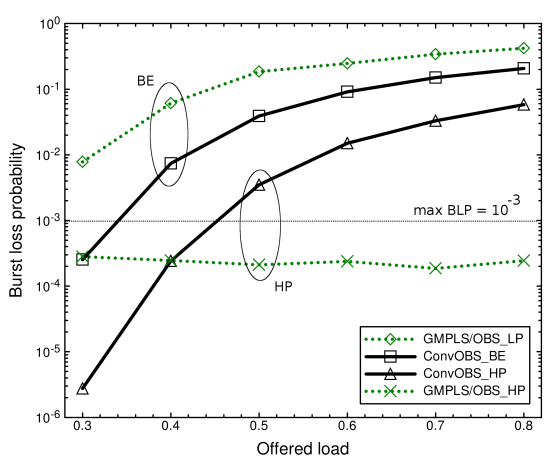

Fig. 4. Network BLP: NSFNET

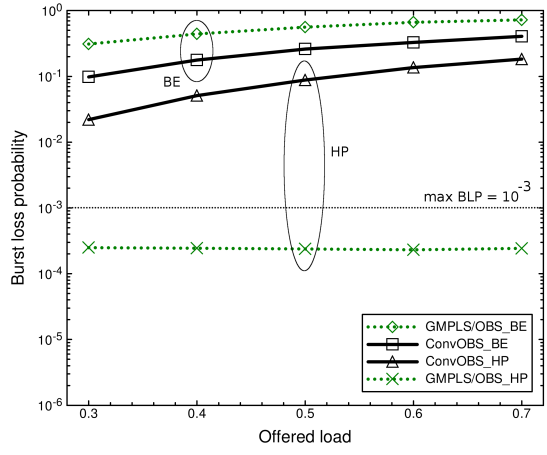

Fig. 5. Network BLP: EON

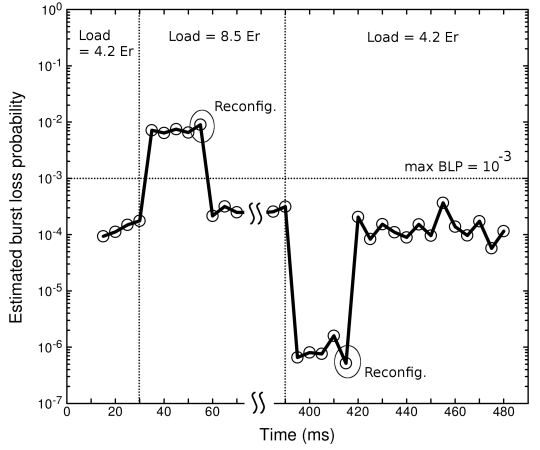

Fig. 6. Monitoring results tioning, however, that long-term changes on the traffic matrix would eventually require a reconfiguration of the VT.

\section{CONCLUSION}

This paper introduced a GMPLS-enabled OBS network architecture providing QoS-aware burst transport services. Aiming to assure the QoS requirements for the high-priority service class, a MILP formulation was presented to compute an optimal virtual topology of burst LSPs over the OBS data plane. In this context, extensions to the standard GMPLS protocols in order to set-up and dynamically reconfigure the computed virtual topology were also proposed. Extensive simulations results highlighted that the proposed virtual topology design allows guaranteeing absolute BLP figures for HP class, even in high load situations. Furthermore, the proposed dynamic burst LSP reconfiguration mechanisms yielded an efficient adaptation to those unexpected traffic surges, keeping the burst loss probability of the high-priority traffic below the requested maximum values.

\section{ACKNOWLEDGMENT}

The authors would like to thank their colleague Fernando Agraz for his valuable comments on the paper. This work has been supported by the Portuguese Government Entity, Fundação para a Ciência e a Tecnologia (FCT), through the grant SFRH / BD / 36950 / 2007, by the Spanish Ministry of Science and Innovation through the "COPERNic" project (TEC2009-13252), the Catalan Government under the contract SGR-1140, and the Polish Ministry of Science and Higher Education under the contract 643/N-COST/2010/0..

\section{REFERENCES}

[1] S.J.B. Yoo, "Optical Packet and Burst Switching Technologies for the Future Photonic Internet", IEEE/OSA J. Lightw. Technol., Dec. 2006.

[2] C. Qiao, M. Yoo, "Optical burst switching - A new paradigm for an optical Internet", J. High Speed Networks, vol. 8, no. 1, Jan. 1999.

[3] J. Hwang, M. Yoo, "Congestion control algorithm based on prediction for OBS networks", in Proc. COIN-NGNCON 2006, Jul. 2006.
[4] J. Liu, N. Ansari, T. J. Ott, "FRR for latency reduction and QoS provisioning in OBS networks", IEEE J. Sel. Areas Commun., vol. 21, no. 7, pp. 1210-1210, Sep. 2003.

[5] Q. Chen, G. Mohan, K.-C. Chua, "Route optimization in optical burst switched networks considering the streamline effect", Computer Networks, vol. 52, no. 10, pp. 2033-2044, Jul. 2008.

[6] Li Yang, G.N. Rouskas, "Adaptive path selection in OBS networks", IEEE/OSA J. Lightw. Technol., vol. 24, no. 8, pp. 3002-3011, Aug. 2006.

[7] A. Pantaleo, M. Tornatore, A. Pattavina, C. Raffaelli, F. Callegati, "Dynamic service differentiation in OBS networks", in Proc. Broadnets 2007, pp. 37.39, Sep. 2004.

[8] F. Callegati, W. Cerroni, C. Raffaelli, P. Zaffoni, "Wavelength and time domain exploitation for QoS management in optical packet switches", Computer Networks, vol. 44, no. 4, pp. 569-582, Mar. 2004.

[9] A. Jajszczyk, "Automatically Switched Optical Networks: Benefits And Requirements", IEEE Commun. Mag., Feb. 2005.

[10] A. Farrel, I. Bryskin, "GMPLS: architecture and applications", Morgan Kaufmann Series in Networking, 2006.

[11] L. Berger, "Generalized Multi-Protocol Label Switching (GMPLS) Signaling Resource ReserVation Protocol-Traffic Engineering (RSVP-TE) Extensions", IETF RFC 3473, Jan. 2003.

[12] K. Kompella, Y. Rekhter, "Signaling Unnumbered Links in RSVP-TE", IETF RFC 3477, Jan. 2003.

[13] M. Klinkowski et al., Graphs and Algorithms in Communication Networks - Studies in Broadband, Optical, Wireless, and Ad Hoc Networks. pp. 165-181, (ed.) A. Koster and X. Muñoz, Springer-Verlag, 2009, ISBN 978-3-642-02249-4.

[14] Z. Rosberg, H. L. Vu, M. Zukerman, J. White, "Performance Analyses of Optical Burst Switching Networks", IEEE J. Sel. Areas in Commun., vol. 21, no. 7, Sep. 2003, pp. 1187-1197.

[15] M. Klinkowski et al., "Virtual Topology Design in OBS Networks", in Proc. IEEE ICTON2010, Munich, Germany, Jun. 2010.

[16] R. Syski, Introduction to Congestion Theory in Telephone Systems, North-Holland, 1960.

[17] M. Minoux, Mathematical Programming: Theory and Algorithms, John Wiley and Sons, 1986.

[18] M. Pióro and D. Medhi, Routing, Flow, and Capacity Design in Communication and Computer Networks, Morgan Kaufmann, 2004.

[19] R. Braden, L. Zhang, "Resource ReSerVation Protocol (RSVP) - Version 1 Message Processing Rules", IETF RFC 2209, Sept. 1997.

[20] D. Awduche, L. Berger, D. Gan, T. Li, V. Srinivasan, G. Swallow, "RSVP-TE: Extensions to RSVP for LSP Tunnels", IETF RFC 3209, Dec. 2001.

[21] O. Pedrola, M. Klinkowski, D. Careglio, J. Solé-Pareta, S. Rumley, C. Gaumier, "JAVOBS: a flexible simulator for OBS network architectures", J. Networks, vol. 5, no. 2, pp. 256-264, Feb. 2010. 